\title{
Automation of test case derivation in respect to test purposes
}

\author{
A. Guerrouata and H. Königb \\ aUniversity Otto von Guericke of Magdeburg \\ Dept. of Computer Science \\ P.O. Box 4120, 39016 Magdeburg, Germany \\ Email: guer@cs.uni-magdeburg.de \\ bBrandenburg Technical University of Cottbus \\ Dept. of Computer Science \\ P.O. Box 101344, 03013 Cottbus, Germany \\ Email: koenig@informatik.tu-cottbus.de
}

\begin{abstract}
Test case derivation and test verdicts specification in respect to given test purposes are an important issue in conformance testing. Since test purposes are commonly expressed in an informal way an automation of this process is not possible. In this paper, we show how this process can be automated for restricted classes of test purposes using a knowledge-based approach. Our approach is founded on a partial formalization of the test purposes that permits an automated derivation of test cases and the deduction of test verdicts. The adaptation of test cases to a given test method is also addressed. An example that illustrates the approach is given.
\end{abstract}

\section{Keywords}

Conformance testing, test purposes, test case derivation, knowledge-based systems

\section{INTRODUCTION}

A formal (or informal) specification of a protocol defines conformance requirements on the implementation of the protocol. The conformance between specification and implementation is defined by means of conformance relations that are deduced from the protocol specification. These relations state the requirements which have to be fulfilled by the implementation to be conform to the specification. In order to assess that an implementation is in compliance with its specification, conformance testing is needed. This is carried out by applying a test suite. It is well-known that the classic test suite generation methods for both finite state machines (FSMs) and labelled transition systems (LTSs) generate a huge number of test cases [Brin88, 
Shen90]. They lack clearly identified test purposes and mostly require a complete specification. The execution of the test cases should improve the likelihood of interworking and interoperability between systems developed by different vendors. Economic and practical considerations, however, considerably restrict the number of test cases that can be carried out. Testers work within the constraints of budget and tight time schedules [Lai95]. Since it is impossible to apply every possible test case under a given time constraint, priority has to be given to test the most important and vulnerable parts of the protocol by defining appropriate test purposes.

In practice, an implementation under test (IUT) is often tested on a functional basis. Experienced testers are capable to assess which conformance requirements are more important to test or which functions of the IUT may be likely incorrectly implemented. Appropriate test cases that match with test purposes addressing conformance requirements or given functions have to be deduced. This implies that the conformance requirements referred to by the test purposes are identifiable with those ones described in the formal specification. For each test event a verdict has to be specified to indicate whether the expected outcome indicates a correct or incorrect behavior of the IUT. The derivation of test cases is manually done in practice because test purposes are informally described. Examining the use of test purposes in practice it seems possible to us to partially formalize certain classes of test purposes in order to structure and to classify them in such a way that their identification based on the conformance requirements described by a formal specification becomes feasible. A partially formal definition and identification of test purposes requires the assistance of a human expert. He can be assisted by knowledge-based systems to store his knowledge and to simplify further development steps.

In this paper, we present a knowledge-based approach for automatically deriving test case for a given test purpose. The approach is based on a partial formalization of test purposes that permits an automated identification of test cases and the specification of test verdicts. The adaptation of the test cases to a given test method is also addressed. We use the SELEXPERT tool [Guer95] for the knowledge-based analysis. It has been designed to select test cases based on the estimation of their fault coverage and cost. It has also been applied to select test cases taking into account data flow aspects. In this paper, we want to extend the use of SELEXPERT for the identification of test purposes to derive test case and test verdicts. In addition, the approach provides a more user-friendly utilization in the sense that it gives more freedom to a user to express his test purposes compared to other approaches (e.g. [Robl92]).

The rest of the paper is organized as follows. Section 2 introduces basic elements related to test purposes and describes the problem of the test case derivation w.r.t. test purposes. In Section 3, we give an overview of the principle of the approach and discuss some classes of test purposes. Section 4 presents the principle of test purpose formalization and of test case derivation and test verdicts specification. In Section 5, we describe the knowledge-based procedure to derive a test case for a given test purpose. An example that illustrates the approach is presented. The test case adaptation to a given test method is discussed in Section 6 . Finally, some concluding remarks are given. 


\section{PRELIMINARIES}

\subsection{Basic concepts}

In the ISO conformance test methodology ISO9646 [ISO91] test cases are organized in a hierarchical structure in which a test suite represents the top level of this hierarchy. Each test case belongs to a precise test purpose. Test purposes are defined informally. There exists no formally defined relationship between test purposes and test cases. Therefore, it is very difficult to exploit test purposes for an automatic derivation of test cases.

A test purpose is a prose description of a narrowly defined objective of testing focusing on a single conformance requirement as specified in the protocol specification. In the context of FMCT [FMCT95], the set of test purposes TP is defined as a finite subset of the set of conformance requirements $R$.

Conformance requirements are the requirements, a conforming implementation has to fulfill. They are divided in two groups: static conformance requirements and dynamic conformance requirements. The first ones represent requirements on possible combinations of values of the formal parameters of the specification whereas the second ones define requirements on the observable behavior of the implementation. In FMCT, the dynamic conformance requirements are defined as requirements implied by the formal specification and the implementation relations. A specification $S$ is expressed as a set of conformance requirements $R_{S}=\left\{r_{1}, r_{2}, \ldots, r_{n}\right\}$. An element $r_{i} \in R_{S}$ represents a single conformance requirement that may be addressed by a test purpose. An implementation $I$ dynamically conforms to a specification $S$ if $I$ satisfies all conformance requirements in $R_{S}$. This is referred as satisfaction relation and denoted by sat: $\left(\forall r_{i} \in R_{S}: I\right.$ sat $\left.r_{i}\right)$.

In this paper, the meaning of conformance is implicitly based on this type of relation since the associated verdicts are essentially based on the fulfillment of the conformance requirements by the IUT as indicated by the test purposes. In addition, we confine ourselves to dynamic conformance requirements, since these represent the main part of a protocol.

A test case refers to a given test architecture [ISO91] and consists of

- a test purpose

- a preamble

a test body

- test verdicts.

A preamble is a sequence of events that transfers the IUT into a desired state from which the test body will start. A test body consists of sequences of test events according to the test purpose. A test verdict indicates the conformance of the IUT with respect to the test purpose. The verdict pass is assigned to the outcome of a test event if it matches the test purpose, otherwise the verdict fail. If no clear statement on conformance the verdict inconclusive is associated. The postamble is optional [ISO91], therefore, it is not considered here.

\subsection{Description of the problem}

Let be given a set of test purposes $T P$, and a test architecture $T A$. To derive a test case $T C_{i}$ for a given test purpose the following 5-tuple $T C_{i}=\left\langle T P_{i}, T A_{i}, P A_{i}, T B_{i}, V_{i}\right\rangle$ has to be determined in 
which $T P_{i} \in T P$ denotes the test purpose corresponding to a conformance requirement $r_{j} \in R_{S}$, $T A_{i}$ the test architecture, $P A_{i}$ the preamble, $T B_{i}$ the test body, and $V_{i}$ the test verdicts. The main issue to automate the derivation procedure consists in mapping a given test purpose to the respective conformance requirements $r_{j} \in R_{S}$ in the specification. Unfortunately, this problem cannot be generally solved unless the set of test purposes $T P$ is reasonably restricted and adequately formalized. This is due to the fact that test purposes are usually informal and may express different kinds of conformance requirements, e.g. requirements on the dynamic behavior, requirements referring to the test context and so on.

\section{PRINCIPLE OF THE APPROACH}

In this section, we first give a brief overview of our approach to automate the test case derivation w.r.t. test purposes. Then, we examine certain classes of test purposes to illustrate the principle of the approach. We discuss the possibility of their formalization and the feasibility of an automatic identification of test purposes when these are restricted to some 'typical' classes.

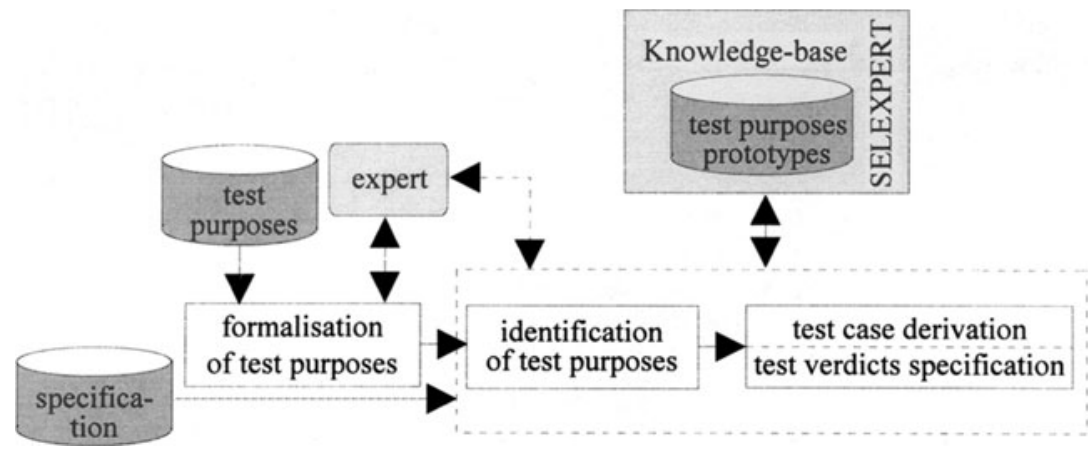

Figure 1 Overview of the approach.

The principle of our approach is depicted in Figure 1. The approach is based on the assumption that test purposes can be partially formalized and classified related to their syntax and objectives as will be discussed below. A prototype of each class is stored as a fact in the knowledge-base of SELEXPERT. It describes the syntactical basic structure of the test purposes of this class and the conformance requirements they are related to. With each class, an algorithm is associated for deriving the test case from the specification presented as behavior tree and for specifying the verdicts. The behavior tree is supposed to be deterministic. It can be obtained, for instance, by simulation [Guer95, Ulri93]. If such a knowledge-base is set up SELEXPERT can be used to automatically derive test cases and test verdicts from a given set of test purposes in connection with the respective formal description. The derivation procedure may be supported by a human expert. The assistance of the human expert is needed if the automatic identification of a test purpose fails, i.e. no test purpose 
prototype matches the given one. In this case, a new prototype may be added to the knowledge-base which is thus stepwise extended.

Our approach to formalize test purposes was inspired by considering representations of test purposes in practice and literature. We give some examples here.

First, we present some test purposes used in a conformance test center for testing Profibus protocols. Profibus (process field bus) [DIN91] is the German fieldbus system used for process-oriented applications in the automation technique area. The testing comprises among other the fieldbus message specification (FMS) using the remote test method [ISO91]. FMS represents a sublayer of the application layer. The conformance testing takes place by applying test cases such as those given in Table 1.

Table 1 Test purposes for FMS of Profibus

\begin{tabular}{ll}
\hline Test case identifier & Test purpose \\
\hline satv0100 & $\begin{array}{l}\text { send write-with-type-request to IUT and await } \\
\text { write-with-type-response from IUT }\end{array}$ \\
sciy1100 & send initiate-request to IUT and await abort-request from IUT \\
sciv9100 & send initiate-request to IUT and await initiate-confirmation from \\
& IUT \\
\hline
\end{tabular}

The words in italic of the test purposes in Table 1 denote the data concerned by the test purpose. The other words can be considered as keywords that characterize the test purpose. The keywords possess certain semantics in the sense that they, for instance, indicate an order in which the interactions, i.e. the data, must occur. Because of the simplicity of the representation in which these test purposes are expressed, it should be possible to define a formal structure for such a type of test purposes. This formal structure can be used as a prototype of the test purposes to automatically identify test purposes that follow the same structure. Thus, the identification of the conformance requirements addressed by the test purpose with corresponding ones described in the specification can be automated to derive the test case and test verdicts.

In [Brin91], a notation (after-must) based on the LTS formalism was introduced to express test purposes. This notation is used in the form: (after $\sigma$ must $A$ ). $\sigma$ denotes an event sequence, i.e. a trace, leading to a given state, and $A \subseteq L$ a subset of the label set $L$ of the LTS. $A$ represents a set of events that may be performed by the given process. If $A$ is restricted to one element, i.e. an event, the test purpose addresses a single conformance requirement in this case. If test purposes are supposed to be expressed in this notation, they can be identified by the keywords after and must, and by exploiting the indicated path $\sigma$ that refers to the given conformance requirement included in $A$. Furthermore, this notation may be enriched to become more familiar for an ordinary user, as in the example: "after $\sigma$ the reaction of IUT must $A^{\prime \prime}$ and so on.

In other works, especially those exploiting the structure information of formal specifications (e.g. [Ulri93, Velt92]), test purposes generally concern a process or a module of the specification as in the example: "check the correct execution of process connection_request". A process or a module describes a function of the specified system in this case. Since these 
representations are not very complex, it is possible to define a formal structure for such a class of test purposes. However, this supposes that the specification may be represented in a suitable form, i.e. as an hierarchy of processes.

\section{FORMALIZATION OF TEST PURPOSES}

In this section, we show how formal structures can be defined for different classes of test purposes. For lack of space, we discuss here only some examples to demonstrate the principle.

There exist two types of test purposes:

- test purposes concerning static conformance requirements (not considered here as mentioned previously);

- test purposes referring to dynamic conformance requirements focusing in general on a certain property of the specified system (e.g. a function), or on a single conformance requirement, as in the example: "after receiving event 1 the IUT must respond with event 2 ". A conformance requirement may also concern timing aspects as, for instance, "test whether event is returned within time limit of $n$ (seconds)". The timing aspect depends the given test architecture and particularly refers to the propagation delays of messages and so on [Chan92].

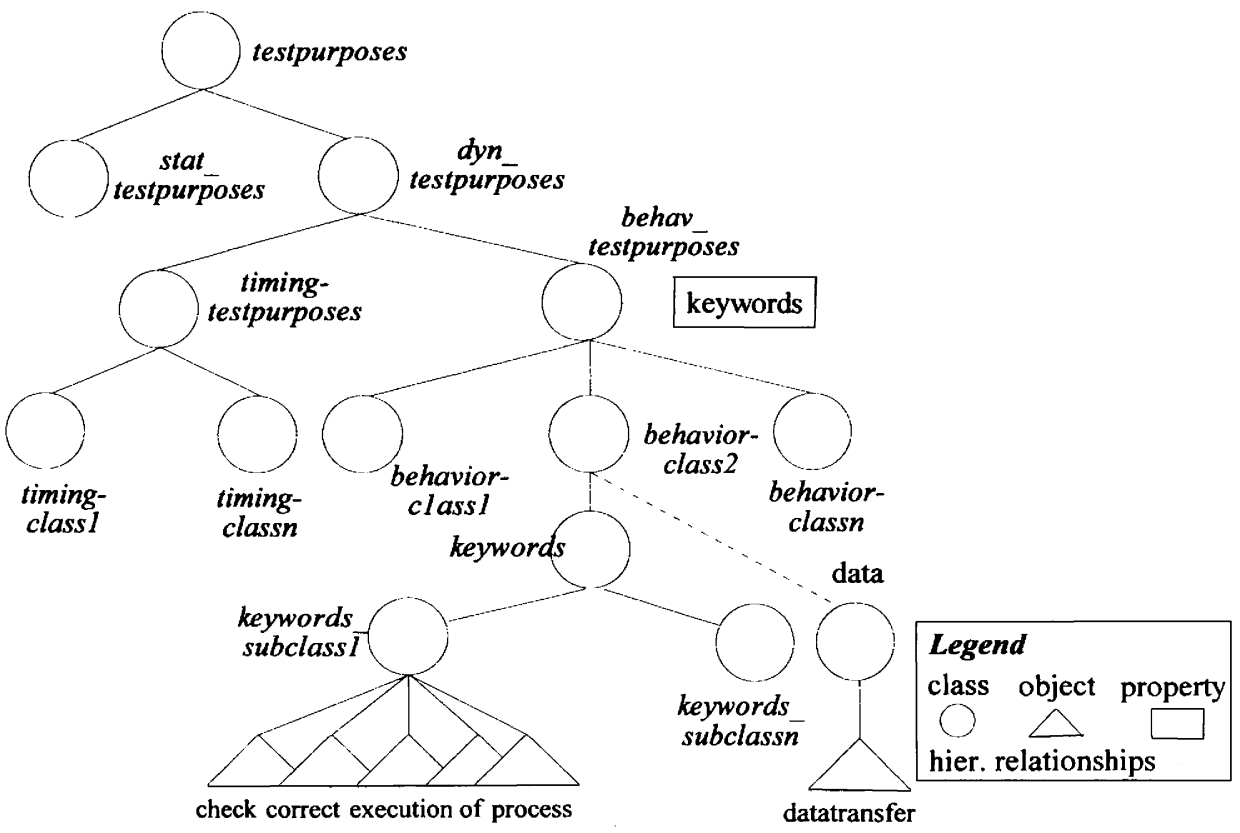

Figure 2 Knowledge representation of test purposes. 
The knowledge representation formalism used in SELEXPERT is the object paradigm [Rumb91]. Figure 2 shows the representation of test purposes using this paradigm. The two types of test purposes indicated above constitute classes, noted as stat_testpurposes and dyn_testpurposes, respectively. They are subclasses of a superclass testpurposes that comprises all test purposes. Dyn_testpurposes is again organized into two other subclasses: behav_testpurposes and timing_testpurposes. These subclasses comprise test purposes concerning the behavior and the timing aspect, respectively. Each subclass is characterized by keywords that represent a property in respect to the object paradigm. The hierarchical structure of the test purposes facilitates the exploration of the different subclasses when identifying a given test purpose. Some of these subclasses will be described in the rest of this section. For convenience, we adopt a Backus-Naur form (BNF) like-notation for describing the formal structure of a class of test purposes, as in the following case:

\section{$\langle$ dyn_testpurposes $\rangle \rightarrow\langle$ behav_testpurposes $\rangle \mid\langle$ timing_testpurposes $\rangle$}

To derive an appropriate test case in respect to a given test purpose, we assume that the specification is given as a finite behavior tree ${ }^{*}$, i.e. a finite set of traces. It can be obtained, for instance, by simulation [Ulri93, Guer95]. A test case consists of a set of traces.

\subsection{Test purposes related to behavior aspects}

To demonstrate the principle of test case derivation and test verdicts specification we consider here two example subclasses of test purposes, denoted by behaviorclass 1 and behaviorclass 2 . They are principally distinguished with respect to their objective and syntax. They belong to class behav_testpurposes indicated above.

\section{Behaviorclass 1}

This subclass of test purposes is supposed to have the following syntax:

$$
\langle\text { behaviorclass } 1\rangle \rightarrow\langle\text { keyword } 1\rangle(\langle\text { event } 1\rangle)\langle\text { keyword } 2\rangle(\langle\text { event } 2\rangle)
$$

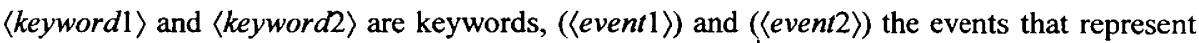
the conformance requirement addressed by the test purpose ${ }^{\dagger}$. An example for this test purpose class would be "after receiving (ICONreq) the IUT must accept (ICONconf)".

A test purpose that can be expressed in this form does not explicitly indicate the localization of the conformance requirement referred to in the specification. An event sequence leading to the given conformance requirement has to be determined in the specification such that its length is minimal. It constitutes the preamble of the test case.

The fact that the event sequence to be adopted as preamble has to be minimal permits us to find the conformance requirement given in the test purpose with minimal effort, i.e. with relatively low cost. In fact, a conformance requirement usually may occur at different places, i.e. branches, of the behavior tree of the specification. This is due to the recursive character of protocols, e.g. through the process calls (see e.g. [Ulri93]). Thus, a given conformance re-

\footnotetext{
"We especially address LOTOS specifications here, but also specifications in other formal descriptions techniques whose semantics can be expressed as behavior trees.

${ }^{\dagger}$ The parentheses aim at distinguishing the data concerned by the test purpose.
} 
quirement can be found in different ways, i.e. event sequences. The optimal event sequence has to be selected in our case to be considered as preamble.

Now, we show how the different components of a test case $T C_{i}$ corresponding to a test purpose $T P_{i}$ in respect to the structure given above, can be derived.

The preamble $P A_{i}\left(T C_{i}\right)$ consists of only one event sequence that can be obtained as follows:

$$
P A_{i}\left(T C_{i}\right)=\sigma_{i} \in S \text { with }\left(\sigma_{i}=s_{0}-e_{i 1} \rightarrow s_{i 1}-e_{i 2} \rightarrow s_{i 2} \ldots s_{i p-1}-e_{i p} \rightarrow s\right)
$$

with $\left(s_{i} e \rightarrow s_{j}\right)$ denotes a transition $t$ where $s_{i}$ represents its current state, $e$ an event, and $s_{j}$ the tail state of $t$

such that

- $s_{0}$ is the initial state of the specification $S$;

- $\left(\exists \sigma^{\prime} \in S\right)$ such that $\left(\sigma^{\prime}=s_{0}-e_{i 1} \rightarrow s_{i 1}-e_{i 2} \rightarrow s_{i 2} \ldots s_{i p-1}-e_{i p} \rightarrow s-\langle\text { event } 1\rangle \rightarrow s\right)^{\ddagger}$;

- length $\left(\sigma_{i}\right) \leq \operatorname{length}\left(\sigma_{j}\right)$ for all $j \neq i$, with $\sigma_{j} \in S$ and $\left(\sigma_{j}=s_{0}-e_{j 1} \rightarrow s_{j 1}-e_{j 2} \rightarrow s_{j 2} \ldots s_{j q-1} \longrightarrow e_{j q} \rightarrow s_{j q}\right)$ such that

$\sigma_{j}^{\prime} \in S$ with $\left(\sigma_{j}^{\prime}=s_{0}-e_{j 1} \rightarrow s_{j 1}-e_{j 2} \rightarrow s_{j 2} \ldots s_{j q-1}-e_{j q} \rightarrow s_{j q}-\langle\right.$ event 1$\left.\rangle \rightarrow s^{\prime \prime}\right)$.

Note that for convenience, in the event sequences above states are explicitly represented. Furthermore, 〈event 1$\rangle$ refers to the event indicated in the test purpose and length (...) denotes the number of events of the given event sequence. We have omitted, to explicitly represent the gates associated to the events in the test sequences given above for more abstraction at this level.

The test body represents a set of event sequences that can be obtained in relation to the preamble determined above and the test purpose as follows:

$T B_{i}\left(T C_{i}\right)=\left\{\sigma_{i} \in S \mid \sigma_{i}=s_{i 0}-e_{i 1} \rightarrow s_{i 1}-e_{i 2} \rightarrow s_{i 2} \ldots s_{i m-1}-e_{i m} \rightarrow s_{i m}\right\}$

such that

- $\left(\forall \sigma_{i} \in T B_{i}\left(T C_{i}\right)\right)\left(\left(s_{i 0} \equiv s\right)\right.$ and $\left(e_{i 1}=\langle\right.$ event 1$\left.\left.\rangle\right)\right)$;

- $\left(\exists \sigma_{k} \in T B_{i}\left(T C_{i}\right)\right)$ with $\left(\sigma_{k}=s_{k 0}-e_{k 1} \rightarrow s_{k 1}-e_{k 2} \rightarrow s_{k 2} \ldots s_{k m-1}-e_{k m} \rightarrow s_{k m}\right)$ such that $\left(e_{k 2}=\langle\right.$ event 2$\left.\rangle\right)$.

Note $\langle$ event 1$\rangle$ and $\langle$ event 2$\rangle$ indicated above refer to the events addressed by the test purpose, and $s$ refers to the state indicated in the preamble $P A_{i}\left(T C_{i}\right)$.

To specify the verdicts $V_{i}\left(T C_{i}\right)$ w.r.t. the test purpose $T P_{i}\left(T C_{i}\right)$ for the test body $T B_{i}\left(T C_{i}\right)$ an algorithm VerdictBehavClass 1 is applied [Guer96]. It has as input the test body $T B_{i}\left(T C_{i}\right)$, the test purpose $T P_{i}\left(T C_{i}\right)$, the behavior tree of the specification $S$, and the test architecture $T A_{i}$. The output of the algorithm are the test verdicts $V_{i}\left(T C_{i}\right)$ that are specified for the test body $T B_{i}\left(T C_{i}\right)$. The algorithm first determines the conformance requirement addressed by the test purpose. This is facilitated by the derived preamble $P A_{i}\left(T C_{i}\right)$. Then the corresponding verdicts are associated by considering the possible interactions of the IUT at the different PCOs (points of control and observation) of $T A_{i}$ in relation to the test purpose and the specification.

\footnotetext{
${ }^{\ddagger}$ For convenience, the parentheses delimiting the data (i.e. events) in the test purpose are omitted.
} 
According to the object concept the different components of a test case are considered as properties associated with the object $T C_{i}$. For their determination, methods have been attached to these properties. The algorithm VerdictBehavClass 1 , for instance, is attached as a method to the property $V_{i}$ of the object $T C_{i}$.

\section{Behaviorclass2}

Now, we consider the structure of example subclass behaviorclass 2 that address a process of a specification.

\section{$\langle$ behaviorclass 2$\rangle \rightarrow\langle$ keyword 1$\rangle(\langle$ function 1$\rangle)[\langle$ keyword 2$\rangle(\langle$ function 2$\rangle)]$}

The test purpose "check the correct execution of process (wait)" is an example for this class.

( $($ function 1$\rangle)$ indicates a process ${ }^{\S}$ name referred to by the test purpose. (〈function 2$\left.\rangle\right)$ is facultative; it is ignored in the example given above. It represents another process name in which ( $($ function 1$\rangle)$ is defined. This is necessary if the peer entities of a protocol specification may include functions that can be ambiguously referenced.

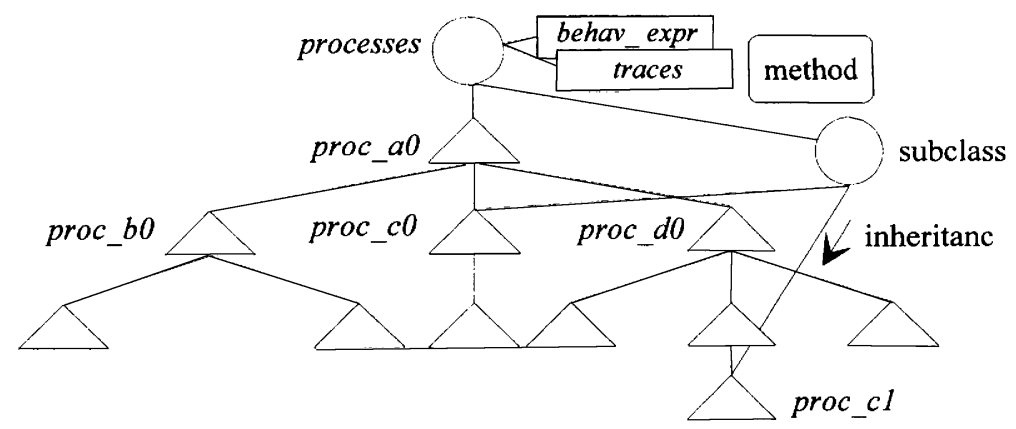

Figure 3 Representation of a LOTOS specification using the object paradigm.

To allow the identification of a process, the specification is represented as an hierarchy of processes (Figure 3). Considering the object paradigm, each (sub)process is viewed as object that is characterized by two main properties: behav_exp and traces. The first includes the definition of the process whereas the second indicates the resulting behavior of behav_exp as a set of traces. The latter is obtained by attaching a method to the property traces. The method calls a simulator to evaluate the given behavior expression behav_exp to generate the behavior tree. The latter represents the behavior described by the specification, if proc_a $a$ is subject of simulation, and a partial behavior if another subprocess is concerned by simulation. The value obtained for the property traces may be automatically inherited by means of the inheritance mechanisms [Rumb91]. Thus, the processes possessing the same definition can be attached to a same subclass. They inherit the same value to the property trace, e.g. proc_co and proc_cl. The representation of a specification as a hierarchy of objects facilitates the exploration of the

\footnotetext{
$\S$ This especially concerns a LOTOS specification.
} 
processes of the specification and therefore, the identification of the one addressed by the test purpose.

The preamble $P A_{i}\left(T C_{i}\right)$ consists of exactly one event sequence, i.e. a trace, that is obtained as follows:

$P A_{i}\left(T C_{i}\right)=\sigma \in \mathrm{S}$ with $\left(\sigma=s_{0}-e_{k 1} \rightarrow s_{k 1} \ldots s_{k r-1}-e_{k r} \rightarrow s_{k r}\right)$

such that

$\left(\forall \sigma_{i}^{\prime} \in P\right)$ where $\left(\sigma_{i}^{\prime}=s_{P 0}-e_{i 1} \rightarrow s_{i 1} \ldots s_{i q-1}-e_{i q} \rightarrow s_{i q}\right)$, implies

- $\left(s_{0}-e_{k 1} \rightarrow s_{k 1} \ldots s_{k r-1}-e_{k r} \rightarrow s_{k r}-e_{i 1} \rightarrow s_{i 1} \ldots s_{i q-1}-e_{i q} \rightarrow s_{i q} \in B_{S}\right)$ with $\left(s_{k r} \equiv s_{P 0}\right)$;

- $\left(\forall \sigma_{j} \in Q\right)\left(\right.$ length $(\sigma) \leq l$ length $\left.\left(\sigma_{j}\right)\right)$.

Note that $B_{S}=\left\{\sigma_{1}, \sigma_{2}, \ldots, \sigma_{n}\right\}$ represents the behavior tree of the specification $S$, where $s_{0}$ denotes its initial state, and $P=\left\{\sigma_{1}^{\prime}, \sigma_{2}^{\prime}, \ldots, \sigma_{m}^{\prime}\right\}$ is the behavior described by the given process. $\sigma_{i}$ denotes a trace, and $\sigma_{i}^{\prime}$ a subsequence of events w.r.t. a corresponding trace of $B_{S}$. $Q$ is a finite set of traces that relates to $P$ and $B_{S}$ as follows:

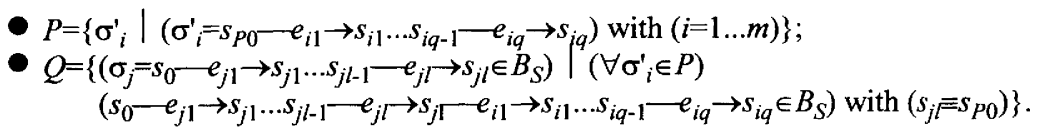

The test body $T B_{i}\left(T C_{i}\right)$ consists of the behavior described by the process that is referred to as ( $($ function 1$\rangle)$ in the test purpose, i.e. $T B_{i}\left(T C_{i}\right)=\left\{\sigma_{1}^{\prime}, \sigma_{2}^{\prime}, \ldots, \sigma_{m}^{\prime}\right\}$. The corresponding verdicts $V_{i}\left(T C_{i}\right)$ can be deduced by applying an appropriate algorithm VerdictBehavClass2 [Guer96] similar to that used for the test purposes subclass behaviorclass 1 . It considers the obtained test body $T B_{i}\left(T C_{i}\right)$ described by the process $P$, the behavior tree of the specification $S$, the test purpose $T P_{i}\left(T C_{i}\right)$, the determined preamble $P A_{i}\left(T C_{i}\right)$, and the test architecture $T A_{i}$. It first identifies the events that represent reactions of the IUT to given events of process $P$. After that, a verdict pass is specified for the reaction in question, if it is described in the process. Otherwise an inconclusive or fail verdict is specified depending on description in the specification of the reaction by considering the different PCOs of the test architecture $T A_{i}$.

\subsection{Test purposes related to timing aspects}

The timing aspect is an important factor in testing. It is especially introduced by the propagation delays and message loss. Propagation delays that are implied by the underlying servicemedium may be the cause that a tester observes test events in an order different from the IUT side. Even though the underlying service is usually supposed to be sufficiently reliable, message delays and message loss may happen in practice which cause the expiration of the respective timer, thus leading to an inconclusive verdict [Chan92].

We again consider two example subclasses of test purposes: timingclass 1 and timingclass 2 . They belong to the test purposes class timing_testpurposes. The reason of distinguishing these subclasses is the same as that indicated in Section 4.1 . 


\section{Timingclass 1}

The test purposes belonging to this subclass are supposed to address conformance requirements without considering their emplacements in the specification as explained above. In other words, the path leading to the concerned conformance requirement is not explicitly given. We consider that the occurrence of the conformance requirement that can be reached with the lowest cost implicitly represents the objective of the test purpose.

$\langle$ timingclass 1$\rangle \rightarrow\langle$ keyword 1$\rangle(\langle$ event $\rangle)\langle$ keyword 2$\rangle(\langle$ timevalue $\rangle)$, e.g.

"check if (ICONconf) is returned within time limit (3)" where the time limit is expressed in a given time unit, e.g. in seconds.

$\langle$ keyword 1$\rangle$ and $\langle$ keyword 2$\rangle$ identify the test purpose. (〈event $\rangle)$ is the event name to which the test purpose is related. ( $\langle$ timevalue $\rangle)$ represents the period in which the IUT must react to the event $(\langle$ event $\rangle)$. After expiring ( $\langle$ timevalue $\rangle)$ a time-out will occur for the timer associated

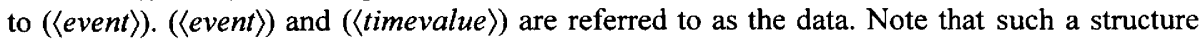
gives no indication about the localization of the test event $(\langle$ event $\rangle)$. The event ( $\langle$ event $\rangle)$ is automatically identified in the protocol specification in such a way that the event sequence leading to it is optimal.

The preamble $P A_{i}\left(T C_{i}\right)$ of the test case $T C_{i}$ consists of exactly one event sequence that is determined as follows:

$P A_{i}\left(T C_{i}\right)=\sigma_{i} \in S$ with $\left(\sigma_{i}=s_{0} \smile e_{i 1} \rightarrow s_{i 1}-e_{i 2} \rightarrow s_{i 2} \ldots s_{i p-1}-e_{i p} \rightarrow s\right)$

such that

- $s_{0}$ represents the initial state of the specification;

- there exists a transition $t\left(t=s_{i p}-e_{i k} \rightarrow s_{i k}\right)$ with $\left(s_{i p}=s\right)$ such that

$$
\left(\sigma_{i}^{\prime}=s_{0} e_{i 1} \rightarrow s_{i 1} \longrightarrow e_{i 2} \rightarrow s_{i 2} \ldots s_{i p-1}-e_{i p} \rightarrow s_{i p}-e_{i k} \rightarrow s_{i k}-\langle e v e n t\rangle \rightarrow s^{\prime} \in S\right)
$$

- length $\left(\sigma_{i}\right) \leq l e n g t h\left(\sigma_{j}\right)$ for all $j \neq i$, with $\left(\sigma_{j}=s_{0}-e_{j 1} \rightarrow s_{j 1} \longrightarrow e_{j 2} \rightarrow s_{j 2} \ldots s_{j q-1} \longrightarrow e_{j q} \rightarrow s_{j q} \in S\right)$ such that

$$
\begin{aligned}
& \circ \quad \exists \text { a transition } t^{\prime}\left(t^{\prime}=s_{j q} \longrightarrow e_{j k} \rightarrow s_{j k}\right) ; \\
& \circ \quad \sigma_{j}^{\prime}=s_{0}-e_{j 1} \rightarrow s_{j 1}-e_{j 2} \rightarrow s_{j 2} \cdots s_{j q-1}-e_{j q} \rightarrow s_{j q}-e_{j k} \rightarrow s_{j k}-\langle\text { event }\rangle \rightarrow s^{\prime \prime} \in S .
\end{aligned}
$$

Note that $\langle$ event $\rangle$ indicated above refers to the test event given in the test purpose.

The test body $T B_{i}\left(T C_{i}\right)$ of the test case $T C_{i}$ consists of a set of event sequences that is obtained in connection with the preamble, the test purpose, and the specification as follows:

$T B_{i}\left(T C_{i}\right)=\left\{\sigma_{j} \in S \mid\left(\sigma_{j}=s_{j 0}-e_{j 1} \rightarrow s_{j 1}-e_{j 2} \rightarrow s_{j 2} \ldots s_{j m-1}-e_{j m} \rightarrow s_{j m}\right)\right\}$ such that $\forall \sigma_{j} \in T B_{i}\left(T C_{i}\right)\left(s_{j 0} \equiv s\right)$

Note that $s$ above refers to the state indicated in the preamble $P A_{i}\left(T C_{i}\right)$ and $m$ represents the length of the given event sequence.

As for the behavior classes we apply an appropriate algorithm VerdictTimingClass 1 [Guer96] to deduce the verdicts $V_{i}\left(T C_{i}\right)$ for the derived test body $T B_{i}\left(T C_{i}\right)$ by taking into con- 
sideration the preamble $P A_{i}\left(T C_{i}\right)$, the test purpose $T P_{i}\left(T C_{i}\right)$, the specification $S$ and the testing architecture $T A_{i}$. The algorithm first determines an event in the specification for which $(\langle$ event $\rangle)$ addressed by the test purpose represents a reaction. Then a timer whose value is indicated in the test purpose is associated to the given event. After that, the possible verdicts in respect to the time value indicated in the test purpose are specified. Particularly, verdicts depending on the occurrence of the time-out are assigned to ( $\langle$ event $\rangle)$. All possible interactions of the IUT at the different PCOs of the testing architecture are considered.

\section{Timingclass 2}

Finally, we consider another test purposes subclass timingclass 2 . This subclass supposes in contrast to the subclass timingclass 2 that the path leading to the conformance requirement addressed by the test purposes is explicitly given. This is used to deduce the corresponding preamble.

\section{$\langle$ timingclass 2$\rangle \rightarrow\langle$ keyword 1$\rangle(\langle$ eventsequence $\rangle)\langle$ keyword 2$\rangle\langle(\langle$ event $\rangle)\langle$ keyword 3$\rangle(\langle$ timevalue $\rangle)$,}

An example of this subclass would be: "check if after (ICONreq; ICONind; ICONresp; ICONconf; IDISreq; IDISind; IDISreq; ICONreq) the event (ICONind) is returned within time limit (5)".

( $\langle$ eventsequence $\rangle$ ) represents a sequence of events leading to the event $(\langle e v e n t\rangle)$ to which the test purpose is related. It will be exploited to deduce the preamble. (〈timevalue $\rangle)$ is the period within which the IUT has to react to the event ( $\langle$ event $\rangle)$ as explained for timingclass 1 . This subclass is characterized by the fact that a trace ( $($ eventsequence $\rangle)$ is explicitly given which leads to the test event $(\langle$ event $\rangle)$ concerned by the test purpose. This is relevant when a particular attention is accorded to a precise occurrence of the given event.

In the manner of subclass timingclass 1 , we can deduce the various components of a test case $T C_{i}$ in respect to test purposes following the structure of timingclass 2.

\section{TEST CASE DERIVATION PROCEDURE}

In this section, we present the test case derivation procedure for a given test purpose. It is represented in Figure 4.

The procedure starts with the identification of the test purpose. It supposes that the test purpose belongs to a certain prototype class which defines its formal structure as described in Section 4. The variety of instances of a class of test purposes corresponds to the different ways to express a given type of test purposes. This can be done by inserting different syntax units, i.e. words, supposed to be less relevant than the keywords on which the definition of the test purpose is based.

After parsing the test purpose by means of a specific parser routine, the keywords as well as the data concerned by the test purpose are identified. To recognize the objective of the test purpose the keywords are compared with the test purpose prototypes stored as classes in the knowledge base. This operation can be assisted by a human expert if the automatic recognition of the test purpose fails. In this case, the test purpose may be possibly reformulated with the help of an expert and then added to the knowledge base as a new prototype. The identification of a test purpose allows to deduce an appropriate test case and test verdicts. This consists of the determination of the properties preamble, test body and test 
verdicts. Methods that are attached to them are executed each time the identification of a test purpose is carried out.

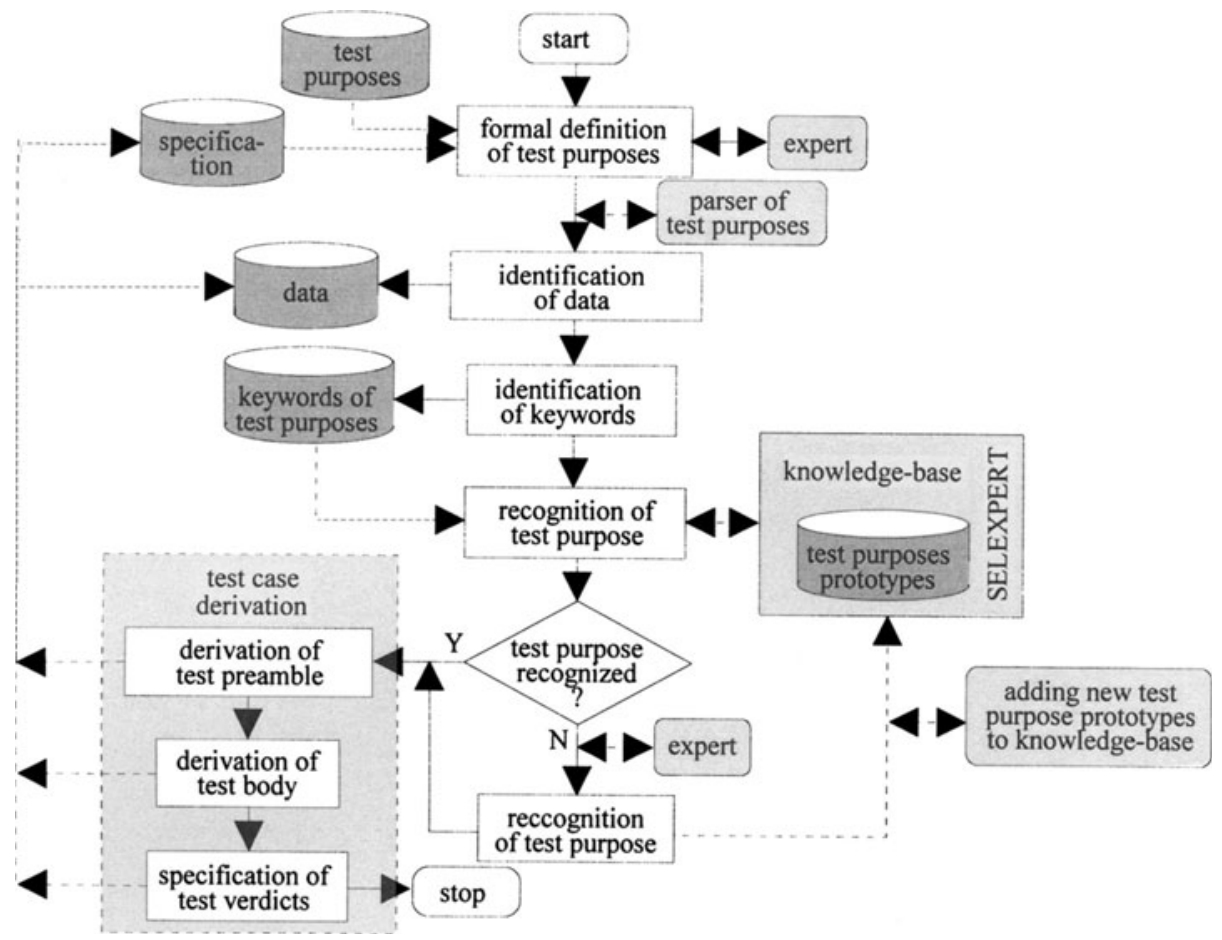

Figure 4 Procedure of the test purpose identification and test case derivation.

Note that the role of an expert consists in assisting the definition of the test purposes and supporting their identification in certain cases. The cooperation of the knowledge engineer and the expert will help to formalize these test purposes, i.e., to assign the respective formal structure that can be used as test purpose prototypes when identifying new test purposes.

\section{Example}

We illustrate the principle of the approach for the INRES protocol [Hogr92]. The test purpose $T P$ is defined as follows: "check the correct execution of process (datatransfer)". datatransfer is supposed to represent the data transfer phase that is specified as a process. The formal structure of this test purpose corresponds to that associated to the subclass behaviorclass 2 (see Section 4). The keywords of an instance of this subclass are supposed to be identifiable with the ones characterizing the given test purpose class. To identify the latter the hierarchy of classes consisting of the different subclasses (behaviorclass 1 , behaviorclass 2 , etc.) has to be explored (see Figure 2). A formal structure as indicated in Section 4 corresponds to each one of them. To identify the test purpose, the parser has to distinguish the two parts of the test 
purpose: keywords and data (here datatransfer). If the test purpose, i.e. its keywords, coincides with one of the instances (e.g. keywords_subclass1), the next step can start that determines the different components of the test case. Otherwise, the assistance of a human expert becomes indispensable.

We suppose the test purpose $T P(T C)$ concerning the process datatransfer as indicated above. A test case that corresponds with $T P(T C)$ is deduced as follows:

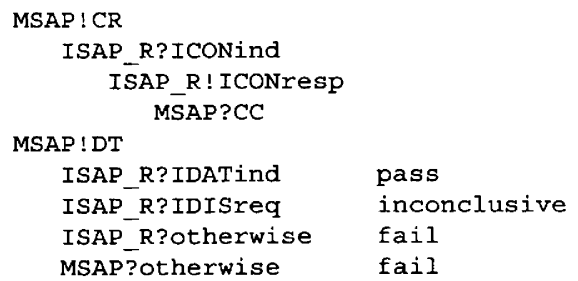

The test event sequence "MSAP!CR . . MSAP?CC" represents the preamble $P A(T C)$ of the test case TC. "MSAP!DT . . MSAP?otherwise" is the test body. The test verdicts $V(T C)$ has been deduced for the test body $T B(T C)$ as explained above. The preamble is optimal in the sense that its length in test events is minimal as indicated in Section 4. The preamble has no verdicts since it is not addressed by the test purpose. Only the behavior addressed by the test purpose was deduced. Note that we have considered here a test architecture whose PCOs are called ISAP R and MSAP situated at the upper and lower tester of the test method, respectively. In addition, otherwise is used to indicate unexpected reactions of the IUT at the PCOs.

\section{TEST CASE ADAPTATION IN RESPECT TO TEST METHODS}

In this section, we describe the procedure to adapt a derived test case to a given test method. This is needed because test cases are usually derived from a formal specification without taking into consideration a given test method.

A specification such as that of an OSI protocol describes the behavior at the service access points. The points where the tester can control and observe the IUT are the PCOs that may not coincide with the service access points belonging to the IUT. The upper tester controls and observes the PCOs connected to the upper access point whereas the lower tester those connected to the lower access point. By varying these aspects different test methods are used, e.g. the local, distributed, coordinated, and remote test methods [ISO91]. In these methods, the lower access point of the IUT is always accessible via an underlying service; the upper access point may be hidden. There exist variants of these methods that can be applied to a multi-layer IUT where an IUT is tested layer by layer. An embedded method is a special case of multilayer testing where one layer can be tested embedded in the other layers.

The principle of test case adaptation that takes a given test method into consideration is illustrated in Figure 5. Starting point is a set of test cases and a test method. These are represented as classes, testcases and testmethods. The class testmethods is characterized by some properties such as identname, uppertester, lowertester, sut, testsystem. Identname indicates the test method name (e.g. DS, distributed single-layer). Uppertester and lowertester contain the PCOs names associated to upper and lower tester, respectively. Sut states the components of the test system. Note that for certain test methods some of these properties are not defined, 
e.g. the value of uppertester is undefined for the coordinated test method. Test coordination procedures have not been considered here for their irrelevance to our purpose.

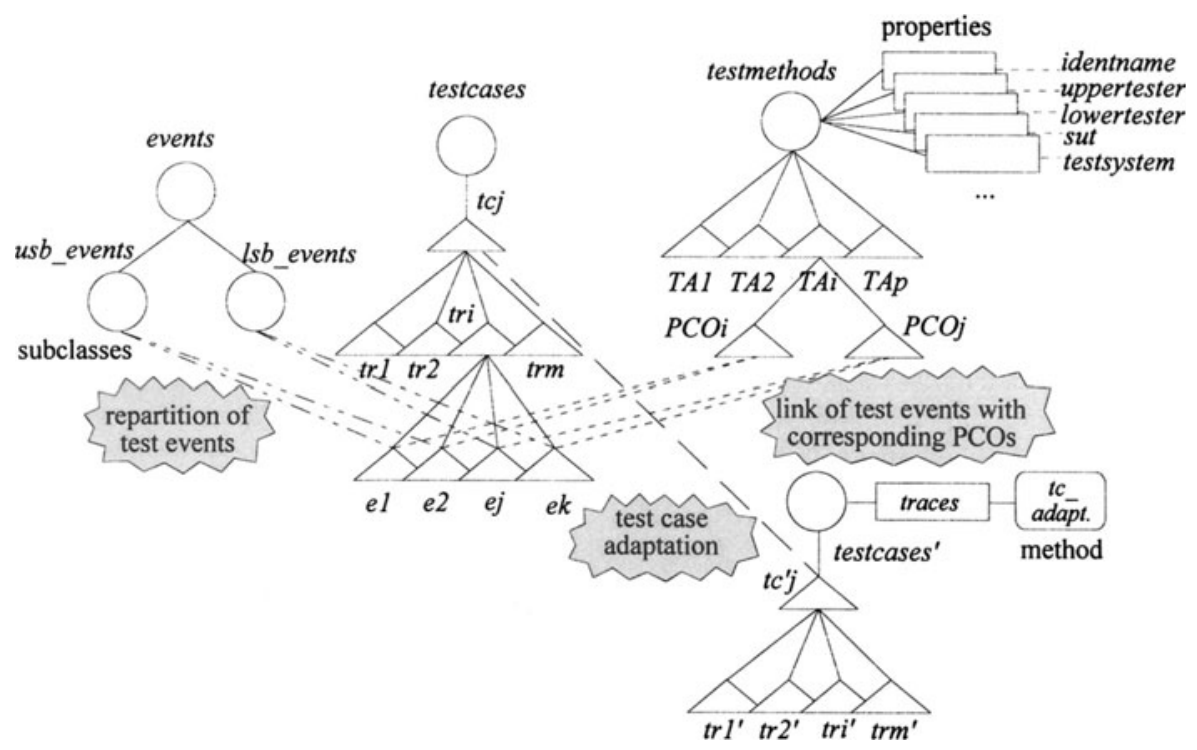

Figure 5 Test case adaptation based on the object paradigm.

There are three main steps for the test case adaptation. First, the test events of the traces of a test case (belonging to the class testcases) are identified. They are linked to the corresponding subclasses, usb_events or lsb_events, according to their occurrence at the upper service boundary or at the lower service boundary of the IUT. The access service boundaries are then linked to their corresponding $P C O_{i}$ that are associated with the upper tester or to the lower tester depending on the given test method $T A_{i}$ (cf. Figure 5). Finally, a class of test cases testcases is deduced. There is a one-to-one relation between its test cases and the input test cases belonging to the class testcases. There is also a one-to-one relation between a trace of a test case of the class testcases' and a trace of a corresponding test case of the class testcases. The property traces is associated to the class testcases' that has to be determined for each member (i.e. a test case) under consideration the given test method. For this purpose, a method is attached to this property to determine the value of the property traces, i.e. to compute all traces composing the given test case. The method implements an algorithm $t c$ adaptation that is not given here.

\section{FINAL REMARKS}

The automation of the test case derivation in respect to given test purposes is a difficult problem. We have shown that this problem can be alleviated by using knowledge-based techniques. Our approach is based on a partial formalization of test purposes. This can be done for important classes of test purposes. The approach was inspired by the experience of 
testers who are often capable to assess what kinds of test purposes are relevant in practice. The cooperation between a knowledge engineer and a human expert in testing may help to formalize and classify test purposes. In an earlier phase of formalization and identification of test purposes, the assistance of a human expert is useful. His support is less needed in later sessions with the progressively growing of the knowledge-base of test purposes prototypes.

Currently, we are extending the approach to cover a large number of real protocols by investigating test purposes prototypes used in these protocols. Furthermore, we plan to make an empirical study of the approach in order to demonstrate the effectiveness of the approach, i.e. to which extent the approach is able to identify test purposes.

\section{REFERENCES}

[Brin88] E. Brinksma. A theory for the derivation of tests. In S. Aggarwal and K. Sabnani, editors, Protocol Specification, Testing, and Verification, VIII, North-Holland, 1988.

[Brin91] E. Brinksma, J. Tretmans, and L. Verhaard. A framework for test case selection. In B. Jonsson, J. Parrow, and B. Pehrson, editors, Protocol Specification, Testing, and Verification, XI, North-Holland, 1991.

[Chan92] S.T. Chanson and Q. Li. On inconclusive verdict in conformance testing. In G.v. Bochmann, R. Dssouli, and A. Das, editors, 5th International Workshop on Protocol Test Systems, North-Holland, 1992.

[DIN91] DIN 19245. Profibus, Process Field Bus. Beuth Verlag GmbH, Berlin, 1991.

[FMCT95] ISO/IEC. Working draft on formal methods in conformance testing. Paris, 1995.

[Guer95] A. Guerrouat, H. König, and A. Ulrich. SELEXPERT - a knowledge-based tool for test case selection. In Proceedings of 8th International Conference on Formal Description Techniques, Montreal, 1995.

[Guer96] A. Guerrouat and H. König. Automatic derivation of test case with respect to test purposes. Technical report, Department of Computer Science, University of Otto von Guericke Magdeburg, Magdeburg, 1996.

[Hogr92] D. Hogrefe. OSI formal specification case study: the INRES protocol and service, revised. Universität Bern, Institut für Informatik, 1992.

[ISO91] Information Technology - Open Systems Interconnection - Conformance testing methodology and framework - Parts 1-5. ISO/IEC 9646, 1991.

[Lai95] R. Lai and W. Leung. Industrial and academic protocol testing: the gap and the means of convergence. Computer Networks and ISDN Systems, 27(1995): 537-547.

[Lunz94] J. Lunze. Künstliche Intelligenz für Ingenieure. Oldenburg Verlag, Munich, 1994 (in German).

[Rob192] T. Robles, J. Manas, and G. Huecas. Specification and derivation of OSI conformance test suites. 3rd LOTOSPHERE-Workshop, Pisa, 1992.

[Rumb91] J. Rumbaugh, M. Blaha, W. Premarlani, F. Eddy, and W. Lorensen. Object-oriented modeling and design. Prentice-Hall, New Jersey, 1991.

[Shen90] Y.-N. Shen, F. Lombardi, and A.T. Dahbura. Protocol conformance testing using multiple UIO sequences. In E. Brinksma, G. Scollo, and C.A. Vissers, editors, Protocol Specification, Testing, and Verification, IX, North-Holland, 1990.

[Ulri93] A. Ulrich and $\mathrm{H}$. König. Test derivation from LOTOS using structure information. In O. Rafiq, editor, 6th International Workshop on Protocol Test Systems, North-Holland, 1993.

[Velt92] R.J. Velthuys, J.M. Schneider, and G. Zörntlein. A test derivation method based on exploiting structure information. In R.J. Linn and M.Ü. Uyar, editors, 2Protocol Specification, Testing and Verification, XII, North-Holland, 1992. 